\title{
THE STRICT TOPOLOGY AND SPACES WITH MIXED TOPOLOGIES
}

\author{
J. B. COOPER ${ }^{1}$
}

ABSTRACT. In a paper about twenty years ago, and later papers, R. C. Buck introduced a new topology, the strict topology, on spaces of continuous functions on locally compact spaces. Since then, a considerable amount of work has been done on these and similar topologies by, among others, Conway, Collins, Rubel and Shields (see references [2], [3], [4], [6], [7], [15]). In the early nineteen-fifties, the Polish mathematicians, Alexiewicz and Semadeni, considered a vector space $E$, on which two norms are defined, and defined a notion of convergence of sequences on $E$ which in some sense mixed the topologies given by the norms ([1] and later papers). In 1957, Wiweger [17] showed that under natural restrictions, the space $E$ could be given a locally convex space structure where convergent sequences were precisely the sequences considered by Alexiewicz and Semadeni. Since then, this method of mixing topologies has been studied and generalised by several mathematicians ([18], [9]).

The purpose of this note is to show that the strict topology for function spaces is a special case of a mixed topology. We then intend to use the theory of mixed topologies to give quick proofs of the basic results on strict topologies. This has the advantage of giving simpler proofs and of eliminating some heavy analysis. It also allows the definition of strict topologies in a more general setting than has been considered until now and in some cases gives new results for the standard situation.

The plan of our paper is as follows: In the first section we state some of the standard definitions and results which will be used in the later sections and we define mixed topologies. The next section is devoted to a development of mixed topologies. The results in this section are essentially due to Alexiewicz, Semadeni, and Wiweger. It has seemed to us worthwhile to give these proofs for the following reasons. Firstly the original proofs are scattered throughout a number of fairly inaccessible papers. Secondly, the conditions we impose on the original topologies are less restrictive than those of the above

Received by the editors July 14, 1969 and, in revised form, June 20, 1970.

AMS 1969 subject classifications. Primary 5420, 5428.

Key words and phrases. Locally convex spaces, two normed spaces, mixed topologies, strict topologies.

1 The author would like to express his gratitude to the Laboratorio de Física e Engenharia Nucleares, Sacavém, Portugal, for the use of an office during the year when this paper was written. 
authors. Thirdly, the hypotheses which we consider have the double advantage of being sufficiently general for any applications and sufficiently strong to allow brief proofs of the results. We note that our treatment has some points in common with a paper of Garling [9].

In the third section we give definitions of function spaces with strict topologies and show that these topologies can be realised as mixed topologies. Finally we apply our results to give quick proofs of the standard results and suggest possible extensions.

The technique of mixing topologies has a number of applications in various branches of analysis, notably summability theory, measure theory on nonlocally compact spaces, and interpolation theorems for analytic functions, and the author hopes to develop these applications in detail in a later paper.

It has been pointed out to the author that some of these results have been obtained independently by J. H. Webb.

During part of the period of preparation of this paper, the author received a grant from the Gulbenkian Foundation in Lisbon.

1. Preliminaries. The terminology in this paper will be the standard terminology of locally convex space theory. We shall use the abbreviation LCS for locally convex space. The following definition is due to Grothendieck. This and the properties of $(D F)$-spaces can be found in reference [10].

Definition 1. A $(D F)$-space is an LCS $E$ which possesses a fundamental sequence $\left(B_{n}\right)$ of bounded sets and has the property that if $\left(U_{n}\right)$ is a sequence of closed, absolutely convex neighbourhoods of zero so that $U=\bigcap_{n=1}^{\infty} U_{n}$ absorbs bounded sets of $E$, then $U$ is also a neighbourhood of zero.

The properties of $(D F)$-spaces which we shall require are the following:

Proposition A (Grothendieck). If $E$ is a $(D F)$-space then

(i) a linear map $u$ from $E$ into an LCS $F$ is continuous iff $u \mid B_{n}$ is continuous for each $n$;

(ii) the strong dual $\left(E^{\prime}, \beta\left(E^{\prime} E\right)\right)$ of $E$ is a Fréchet space.

$(D F)$-spaces are of frequent occurrence in analysis. Important examples are normed spaces and the strong duals of metrisable LCS's.

The following definition is motivated by the behaviour of Fourier series expansions.

Definition 2. A $(C, 1)$ basis for an LCS $E$ is a sequence $\left(x_{n}\right)$ in $E$ with the property that if $x \in E$ there is a unique sequence $\left(\xi_{n}\right)$ of scalars so that the partial sums $s_{n}=\sum_{k=1}^{n} \xi_{k} x_{k}$ converge $(C, 1)$ to $x$. That is, if $\sigma_{n}=(1 / n) \sum_{k=1}^{n} s_{k}$, then $\sigma_{n} \rightarrow x$ in $E$. 
We shall also require the following generalisation of Köthe's completeness theorem:

Proposition B (RAĬKov [13]). Let $E$ be an LCS $E,\left(F_{n}\right)$ a sequence of absolutely convex subsets of $E$ such that

(i) $F_{n}+F_{n} \subseteq F_{n+1}$ for each $n$;

(ii) an absolutely convex subset $V$ of $E$ is a neighbourhood of zero in $E$ iff $V \cap F_{n}$ is a neighbourhood in $F_{n}$ for each $n$;

(iii) each $F_{n}$ is a complete subset of $E$.

Then $E$ is a complete LCS.

A two-normed space is a vector space $E$ on which are defined two norms \|\| and \|\|$*$ so that

(i) $\|x\| * \leqq\|x\|$ for each $x$ in $E$;

(ii) $B=\{x \in E:\|x\| \leqq 1\}$ is closed in the normed space $\left(E,\|\|^{*}\right)$.

Then a sequence $\left(x_{n}\right)$ is said to be $\gamma$-convergent to $x_{0}$ if sup $\left\|x_{n}\right\|<\infty$ and $\left\|x_{n}-x_{0}\right\| * \rightarrow 0$ as $n \rightarrow \infty$. Alexiewicz developed a theory of twonormed spaces using the notion of $\gamma$-convergence of sequences. In the articles [17] and [18] Wiweger showed that $E$ could be given an LCS structure whose convergent sequences were precisely the $\boldsymbol{\gamma}$-convergent sequences. Choose a sequence $\left(U_{n}^{*}\right)$ of absolutely convex neighbourhoods of zero in $\left(E,\|\|^{*}\right)$ and let

$$
U^{\gamma}=\bigcup_{n=1}^{\infty}\left(U_{1}^{*} \cap B+U_{2}^{*} \cap 2 B+\cdots+U_{n}^{*} \cap n B\right) .
$$

Then the set of all such sets $U^{\boldsymbol{r}}$ forms a base of neighbourhoods of zero for the required LCS structure.

We consider the following situation. We have a vector space $E$ with two LCS structures $\tau$ and $\tau^{*}$ satisfying:

(i) $\tau$ is finer than $\tau^{*}$;

(ii) $(E, \tau)$ is a $(D F)$-space with a base $\left(B_{n}\right)$ of absolutely convex bounded sets such that $B_{n}+B_{n} \subseteq B_{n+1}$ for each $n$;

(iii) each $B_{n}$ is $\tau^{*}$-closed.

Then we can define an LCS structure $\gamma=\gamma\left[\tau^{*}, \tau\right]$ on $E$ as follows. Take a sequence $\left(U_{n}^{*}\right)$ of absolutely convex $\tau^{*}$-neighbourhoods of zero and let

$$
U^{\gamma}=\gamma\left(U_{n}^{*}\right)=\bigcup_{n=1}^{\infty}\left(U_{1}^{*} \cap B_{1}+\cdots+U_{n}^{*} \cap B_{n}\right) .
$$

Then the set of all such $U^{\gamma}$ forms a base of neighbourhoods of zero for an LCS topology $\gamma\left[\tau^{*}, \tau\right]$ on $E$.

For future reference we note the following points. The space $E$ has three topological duals, namely $\left(E, \tau^{*}\right)^{\prime},(E, \gamma)^{\prime}$, and $(E, \tau)^{\prime}$ which 
we denote by $E_{1}^{\prime}, E_{2}^{\prime}$, and $E_{3}^{\prime}$ in that order. It follows from Proposition 1 (ii) that $E_{1}^{\prime}$ can be regarded as an (algebraic) subspace of $E_{2}^{\prime}$ which is in turn a subspace of $E_{3}^{\prime}$. The spaces $E_{1}^{\prime}, E_{2}^{\prime}, E_{3}^{\prime}$ will usually be regarded as LCS's with their strong topologies of uniform convergence on the appropriate bounded subsets of $E$.

2. Properties of mixed topologies. The following proposition gives the main properties of $\gamma\left[\tau^{*}, \tau\right]$.

Proposition 1. (i) $\gamma$ is independent of the choice of $\left(B_{n}\right)$;

(ii) $\tau^{*} \subseteq \gamma\left[\tau^{*}, \tau\right] \subseteq \tau$;

(iii) $\tau^{*}=\gamma$ on $\tau$-bounded sets;

(iv) a collection $H$ of linear maps from $E$ into an LCS $F$ is $\gamma$-equicontinuous iff $H \mid B_{n}$ is $\tau^{*}$-equicontinuous for each $n$ : In particular a linear map $u$ from $E$ into $F$ is $\gamma$-continuous if and only if $u \mid B_{n}$ is $\tau^{*}$-continuous for each $n$;

(v) a sequence $\left(x_{n}\right)$ in $E$ is convergent to zero in $\gamma$ iff $\left(x_{n}\right)$ is $\tau$-bounded and $x_{n} \rightarrow 0$ in $\tau^{*}$;

(vi) $\tau$ and $\gamma\left[\tau^{*}, \tau\right]$ have the same bounded sets so that $E_{2}^{\prime}$ is an LCS subspace of $E_{3}^{\prime}$;

(vii) $\tau=\gamma\left[\tau^{*}, \tau\right]$ if $\gamma$ is bornological;

(viii) a set $A \subseteq E$ is $\boldsymbol{\gamma}$-compact iff it is $\tau$-bounded and $\tau^{*}$-compact;

(ix) $\gamma$ is the finest LCS topology on $E$ coinciding with $\tau^{*}$ on $\tau$ bounded sets;

(x) $(E, \gamma)$ is complete iff each $B_{n}$ is $\tau^{*}$-complete;

(xi) if $\tau_{1}^{*}$ and $\tau_{2}^{*}$ are two suitable topologies on $E$, then $\gamma\left[\tau_{1}^{*}, \tau\right]$ $=\gamma\left[\tau_{2}^{*}, \tau\right]$ iff $\tau_{1}^{*}=\tau_{2}^{*}$ on $\tau$-bounded sets;

(xii) $E_{2}^{\prime}$ is the closure of $E_{1}^{\prime}$ in $E_{3}^{\prime}$ (with its strong topology as the dual of $\left.(E, \tau)^{\prime}\right)$ and so is a Fréchet space.

Proof. (iii) Consider a $\tau$-bounded set $B$. We can choose a positive integer $r$ so that $B-B \subseteq B_{r}$. Let $B \cap\left(x_{0}+\gamma\left(U_{n}^{*}\right)\right)$ be a neighbourhood of $x_{0} \in B$ in $\gamma \mid B$ and take $U^{*}=U_{r}^{*}$. Then $U^{*} \cap(B-B) \subseteq U_{r}^{*} \cap B_{r}$ $\subseteq \gamma\left(U_{n}^{*}\right)$. Hence $\left(x_{0}+U^{*}\right) \cap B \subseteq\left(x_{0}+\gamma\left(U_{n}^{*}\right)\right) \cap B$.

(iv) If $H$ is $\gamma$-equicontinuous on $E$, it is certainly $\gamma$-equicontinuous on $\tau$-bounded sets of $E$. By (iii) it is then $\tau^{*}$-equicontinuous on $\tau$-bounded sets.

Conversely, suppose that $H$ is $\tau^{*}$-equicontinuous on each $B_{n}$. Let $W$ be an absolutely convex neighbourhood of zero in $F$ and let $W_{n}=\left(1 / 2^{n}\right) W(n=1,2, \cdots)$. There are $\tau^{*}$-neighbourhoods $\left(U_{n}^{*}\right)$ so that if $u \in H$,

$$
u\left(U_{n}^{*} \cap B_{n}\right) \subseteq W_{n}
$$

Then for any $n, u \in H$, 


$$
u\left(U_{1}^{*} \cap B_{1}+U_{2}^{*} \cap B_{2}+\cdots+U_{n}^{*} \cap B_{n}\right) \subseteq W_{1}+\cdots+W_{n} \subseteq W
$$

and so $u\left(\gamma\left(U_{n}^{*}\right)\right) \subseteq W$ for each $u$ in $H$.

(ii) We show first that $\tau^{*} \subseteq \gamma\left[\tau^{*}, \tau\right]$. Consider the identity map from $\left(E, \gamma\left[\tau^{*}, \tau\right]\right)$ into $\left(E, \tau^{*}\right)$. This is $\tau^{*}$-continuous on $\tau$-bounded sets and so is continuous by (iv).

For the inequality $\gamma\left[\tau^{*}, \tau\right] \subseteq \tau$ we consider the identity map from $(E, \tau)$ into $\left(E, \gamma\left[\tau^{*}, \tau\right]\right)$. Then by (iii) and the fact that $\tau^{*} \subseteq \tau$, this is $\tau$-continuous on $\tau$-bounded sets. Hence it is continuous by Proposition $\mathrm{A}(\mathrm{i})$.

(i) Let $\left(B_{n}\right)$ and $\left(C_{n}\right)$ be appropriate sequences of bounded sets in $E$ and let $\gamma_{1}$ and $\gamma_{2}$ be the corresponding mixed topologies. Now apply (iv) to the identity maps $\left(E, \gamma_{1}\right) \rightarrow\left(E, \gamma_{2}\right)$ and $\left(E, \gamma_{2}\right) \rightarrow\left(E, \gamma_{1}\right)$.

(v) By (iii) we need only show that if $x_{n} \rightarrow 0$ in $\gamma$, then $\left(x_{n}\right)$ is $\tau$-bounded. If this were false, we could find a subsequence $\left(x_{k_{n}}\right)$ so that $x_{k_{n}} \notin B_{n}$. Since $B_{n}$ is $\tau^{*}$-closed we can choose a $\tau^{*}$-neighbourhood $U_{n}^{*}$ so that $x_{k_{n}} \notin B_{n}+2 U_{n}^{*}$ and we can also suppose that $U_{n}^{*}+U_{n}^{*}$ $\subseteq U_{n-1}^{*}(n>1)$. Then for each $n>1$,

$$
\begin{aligned}
U^{\gamma} & =\bigcup_{k=1}^{\infty}\left(U_{1}^{*} \cap B_{1}+\cdots+U_{k}^{*} \cap B_{k}\right) \\
& \subseteq \bigcup_{p=1}^{\infty}\left(\left(B_{1}+\cdots+B_{n-1}+U_{n}^{*}+U_{n+1}^{*}+\cdots+U_{n+p}^{*}\right)\right) \\
& \subseteq B_{n}+2 U_{n}^{*}
\end{aligned}
$$

(recall that $B_{i}+B_{i} \subseteq B_{i+1}$ for each $i$ so that $B_{1}+\cdots+B_{n-1} \subseteq B_{n}$ ). Hence $x_{k_{n}} \notin U^{\gamma}$ for each $n$ which contradicts the fact that $\left(x_{n}\right)$ is a null sequence. This argument is an adaptation of a proof of Wiweger in [17].

(vi) Suppose that $B$ is a $\gamma\left[\tau^{*}, \tau\right]$-bounded set, $\left(x_{n}\right)$ a sequence in $B$, and $\left(\lambda_{n}\right)$ a null sequence of positive scalars. Then $\left(\sqrt{ } \lambda_{n} x_{n}\right)$ is a $\gamma$-null sequence (since $\sqrt{ } \lambda_{n} \rightarrow 0$ ) and so is $\tau$-bounded. Hence $\lambda_{n} x_{n} \rightarrow 0$ in $\tau$. Then $B$ is $\tau$-bounded by a well-known characterisation of bounded sets in LCS's.

(vii) $\mathrm{By}$ (vi), the identity map from $(E, \gamma)$ into $(E, \tau)$ is bounded and so would be continuous if $(E, \gamma)$ were bornological.

(viii) Suppose that $A$ is $\gamma$-compact. Then $A$ is $\gamma$-bounded and so $\tau$-bounded. Then $\tau^{*}=\gamma$ on $A$ and so $A$ is $\tau^{*}$-compact. The converse follows immediately, again from (iii).

(ix) By (iii), $\gamma$ is such a topology. Conversely, if $\gamma^{\prime}$ is an LCS topology agreeing with $\tau^{*}$ on $\tau$-bounded sets, then the identity map from $(E, \boldsymbol{\gamma})$ into $\left(E, \gamma^{\prime}\right)$ is continuous by (iv), i.e. $\boldsymbol{\gamma}$ is finer than $\boldsymbol{\gamma}^{\prime}$. 
(x) This follows from (ix) and Proposition B.

(xi) If $\gamma\left[\tau_{1}^{*}, \tau\right]=\gamma\left[\tau_{2}^{*}, \tau\right]$ then by (iii), $\tau_{1}^{*}=\gamma\left[\tau_{1}^{*}, \tau\right]=\gamma\left[\tau_{2}^{*}, \tau\right]=\tau_{2}^{*}$ on $\tau$-bounded sets. The converse follows from (ix).

(xii) Firstly, $E_{2}^{\prime}$ is an LCS subspace of $E_{3}^{\prime}$ and (iv) implies that it is complete and so closed. We show that $E_{1}^{\prime}$ is dense in $E_{2}^{\prime}$. Let $B$ be a closed absolutely convex $\tau$-bounded set of $E$ and $\epsilon$ a positive number. If $f \in E_{2}^{\prime}$, then by (iv), there is an absolutely convex $\tau^{*}$ neighbourhood of zero $U$ such that $|f(x)|<\epsilon$ if $x \in B \cap U$, i.e. $f \in \epsilon(B \cap U)^{\circ}$ (polar in $E^{*}$, the algebraic dual of $E$ ). But $B^{\circ}+U^{\circ}$ is absolutely convex and $\sigma\left(E^{*}, E\right)$-closed so $(B \cap U)^{\circ}=B^{\circ}+U^{\circ}$. Hence $f \in \epsilon\left(U^{\circ}+B^{\circ}\right)$ and so there is a $g \in \epsilon U^{\circ} \subseteq E_{1}^{\prime}$ such that $f-g \in \epsilon B^{\circ}$, i.e. $|f(x)-g(x)| \leqq \epsilon$ if $x \in B$.

REMARK. The argument used to prove (xii) is taken from the proof of Grothendieck's completeness theorem (see [16, p. 148]).

We now give an example of a natural LCS topology which is a mixed topology. Let $E^{\prime}$ be the dual of a metrisable LCS $E$ and let $\tau=\beta\left(E^{\prime}, E\right), \tau^{*}=\sigma\left(E^{\prime}, E\right)$. Then clearly $\tau$ and $\tau^{*}$ satisfy the conditions for the definition of a mixed topology and we identify $\gamma\left[\tau^{*}, \tau\right]$. (For the case $E$ a normed space, this example has been considered by Wiweger.)

Proposition 2. With the above notation, $\gamma\left[\tau^{*}, \tau\right]=\tau_{c}$, the topology of uniform convergence on the precompact subsets of $E$.

Proof. Consider $i:\left(E^{\prime}, \gamma\right) \rightarrow\left(E^{\prime}, \tau_{c}\right)$. This is continuous by Proposition 1 (iv) since $\sigma\left(E^{\prime}, E\right)$ and $\tau_{c}$ agree on $\beta\left(E^{\prime}, E\right)$-bounded sets by a standard property of equicontinuous sets (see Schaefer $\left[16\right.$, p. 85]). Conversely, $\tau_{c}$ is the finest topology agreeing with $\sigma\left(E^{\prime}, E\right)$ on equicontinuous subsets of $E$ (Banach-Dieudonné theorem) and so is finer than $\gamma$.

Corollary. If $E$ contains $\sigma\left(E^{\prime}, E\right)$-compact sets which are not precompact, then $\gamma$ is not the Mackey topology $\tau\left(E^{\prime}, E\right)$.

3. Spaces with mixed topologies. In this section we consider the two most frequently studied examples of strict topologies and show that these topologies can be obtained as mixed topologies.

Firstly we suppose that $S$ is a locally compact space and that $C(S)$ denotes the vector space of bounded continuous complex-valued functions on $S$. We give $C(S)$ the following LCS structures:

$\sigma$-the Banach space topology defined by the norm

$$
\|f\|=\sup \{|f(s)|: s \in S\} .
$$


$\kappa$ - the topology defined by the family of seminorms

$$
p_{K}(f)=\sup \{|f(s)|: s \in K\}
$$

as $K$ runs through the compacts subsets of $S$.

$\beta$-the topology defined by the family of seminorms

$$
\|f\|_{\phi}=\sup \{|f(s) \phi(s)|: s \in S\}
$$

as $\phi$ runs through the space $C_{0}(S)$ of functions of $C(S)$ which vanish at infinity.

Among the reasons for introducing the topology $\beta$ is that it has the following properties in contrast to $\sigma$. The dual of $(C(S), \beta)$ can be identified with a space of measures on $S$ rather than on the StoneCech compactification of $S$, and the continuous multiplicative functionals on $(C(S), \beta)$ can be identified with $S$ in the usual way. The strict topology was introduced in [2] and has been studied in [5], [6], and [7]. We show now that $\beta$ is the mixed topology defined by $\sigma$ and $\kappa$. This turns out to be surprisingly difficult. We require the following result of Wiweger [18].

Proposition C. Let $\gamma$ be the mixed topology $\gamma\left[\tau^{*}, \tau\right]$ where

(i) the topology $\tau$ is a normed topology defined by the norm $\|$ \| which satisfies the condition that if $x \in E,\|x\|=\sup \left\{p_{\alpha}(x): \alpha \in A\right\}$ where $\left\{p_{\alpha}: \alpha \in A\right\}$ is a family of defining seminorms for $\tau^{*}$;

(ii) if $\left\{p_{n}: n=1,2, \cdots\right\}$ is a subsequence $\left\{p_{\alpha}: \alpha \in A\right\}, x \in E, \epsilon>0$ then for every positive integer $p$, there are vectors $y, z$ of $E$ such that $x=y+z, p_{i}(z)=0(i=1, \cdots, p)$ and $\|y\| \leqq \max \left\{p_{i}(x): i=1, \cdots, p\right\}+\epsilon$. Then the sets of the form $\bigcap_{i=1}^{\infty}\left\{x: p_{i}(x) \leqq \alpha_{i}\right\}$ form a base of neighbourhoods of zero for $\gamma$ as $\left\{p_{i}\right\}$ ranges over the countable subsets of $A$ and $\alpha_{i}$ ranges over all families of positive numbers so that $\alpha_{i} \rightarrow \infty$ as $i \rightarrow \infty$.

We show now that the topologies $\kappa$ and $\sigma$ on $C(S)$ satisfy the conditions of Proposition C. (i) is immediate so we consider (ii). Let $K_{n}$ be a sequence of compact subsets of $S, f$ an element of $C(S)$ and $\epsilon$ a positive number. If $p$ is a positive integer we put $K=\bigcup_{n=1}^{p} K_{n}$. By the uniform continuity of $f$ on $K$ and the local compactness of $S$ we can find a compact set $K^{\prime}$ of $S$ such that $K \subseteq$ int $K^{\prime}$ and $p_{K^{\prime}}(f)$ $\leqq p_{K}(f)+\epsilon$. Let $\phi_{1}, \phi_{2}$ be functions in $C(S)$ so that

(i) $0 \leqq \phi_{i} \leqq 1(i=1,2)$ and $\phi_{1}+\phi_{2}=1$ on $S$;

(ii) $\phi_{1}=1$ on $K$ and $\phi_{1}=0$ on $\mathrm{e} K^{\prime}$;

(iii) $\phi_{2}=0$ on $K$ and $\phi_{2}=1$ on $\mathrm{e} K^{\prime}$.

Then let $g=\phi_{1} f, h=\phi_{2} f$ so that $f=g+h . h(s)=0$ for $s \in K$, i.e. $p_{K_{i}}(h)=0$ $(i=1, \cdots, p)$. Also 


$$
\|g\|=p_{K^{\prime}}(g) \leqq p_{K^{\prime}}(f) \leqq \max \left\{p_{K_{i}}(f): i=1, \cdots, p\right\}+\epsilon .
$$

We can now prove:

Proposition 3. With the above notation, $\beta$ is the mixed topology $\gamma[\kappa, \sigma]$.

Proof. We show first that the identity map $(C(S), \gamma[\kappa, \sigma]) \rightarrow(C(S), \beta)$ is continuous. By Proposition 1 (iv) we need only show that it is $\kappa$ continuous on the set $B_{k}=\{f \in C(S):\|f\| \leqq k\} \quad(k>0)$. Consider $\phi \in C_{0}(S)$ and let $V=\{f \in C(S):\|f \phi\| \leqq \epsilon\}$. Then there is a compact set $K$ such that $|\phi(S)|<\epsilon / 2 k$ if $x \in K$. Let $U=\left\{f \in C(S): p_{K}(f) \leqq \epsilon /\|\phi\|\right\}$. Then $B_{k} \cap U \subseteq V$.

Conversely, consider a $\gamma$-neighbourhood $V$ which, by Proposition C, we can suppose to be of the form $\left\{f \in C(S): p_{n}(f) \leqq \alpha_{n}\right\}$ where for each $n, p_{n}$ is the $\kappa$-seminorm associated with a compact subset $K_{n}$ of $S$. We can further suppose that the sequence $\left(\alpha_{n}\right)$ is strictly increasing and that $K_{n} \subseteq$ int $K_{n+1}$ for each $n$. Then we can construct a $\phi \in C_{0}(S)$ with support contained in $\bigcup_{n=1}^{\infty} K_{n}$ such that $\phi(s)=1 / \alpha_{1}\left(s \in K_{1}\right)$ and $1 / \alpha_{n} \geqq \phi(s) \geqq 1 / \alpha_{n+1}\left(s \in K_{n+1} \backslash K_{n}\right)$. Then if $\|f \phi\| \leqq 1, f \in V$.

We observe now that the space $C(S)$ and its topologies $\kappa$ and $\sigma$ can be defined without requiring that $S$ be locally compact. Also $\kappa$ and $\sigma$ will be such that the mixed topology $\gamma[\kappa, \sigma]$ can be defined. Therefore we make the following

Definition 2. Let $S$ be an arbitrary topological space, $C(S)$ the space of bounded continuous complex-valued functions on $S$. Then the strict topology $\beta$ on $C(S)$ is defined to be the mixed topology.

In the next section we shall use Proposition 1 to show that the known properties of $(C(S), \beta)$ for $S$ locally compact extend to arbitrary $S$.

The second important example of a strict topology is the space $H^{\infty}$ of bounded analytic functions on the unit disc $U=\{z:|z|<1\}$ in the complex plane. We can give $H^{\infty}$ three topologies $\sigma, \kappa, \beta$ in a way similar to $C(S)$. Then as above we can show that $\beta$ is the mixed topology $\gamma[\kappa, \sigma]$. The space $\left(H^{\infty}, \beta\right)$ has been studied in [4], [14], and $[15]$.

4. The spaces $(C(S), \beta)$ and $\left(H^{\infty}, \beta\right)$. We now give a list of properties of $(C(S), \beta)$ and $\left(H^{\infty}, \beta\right)$ which follows from Proposition 1 and the above remarks. These properties are essentially known (for the case $S$ locally compact).

Proposition 4. Let $S$ be a Hausdorff space, $(C(S), \beta)$ as above. Then

(i) $\kappa \subseteq \beta \subseteq \sigma$ and when $S$ is completely regular, $\sigma=\beta$ iff $S$ is compact; 
(ii) $(C(S), \beta)$ is complete if and only if $S$ is such that every complexvalued function on $S$ which is continuous on compact subsets of $S$ and bounded on $S$ is continuous;

(iii) the $\beta$-bounded subsets of $C(S)$ are the uniformly bounded subsets;

(iv) on uniformly bounded subsets of $C(S), \beta=\kappa$;

(v) a sequence $\left(f_{n}\right)$ in $C(S)$ is $\beta$-convergent to zero if and only if it is uniformly bounded and $\kappa$-convergent to zero;

(vi) if $S$ is completely regular, $\beta$ is metrisable iff $S$ is compact;

(vii) a linear map from $C(S)$ into an LCS $F$ is continuous if and only if its restriction to uniformly bounded sets is continuous for $\kappa$;

(viii) $\beta$ is the finest LCS topology on $C(S)$ which agrees with $\kappa$ (or $\beta$ ) on uniformly bounded sets of $C(S)$.

The proof follows immediately from Proposition 1 . We remark that (viii) answers a question posed in [6].

We now turn to $\left(H^{\infty}, \beta\right)$. Firstly, this space has properties (i), (iii), (iv), (v), (vii) of Proposition 4, is complete and is not metrisable. We note also the following points which are special to $\left(H^{\infty}, \beta\right)$.

Proposition 5. (i) Bounded sets of $\left(H^{\infty}, \beta\right)$ are relatively compact. Hence $\left(H^{\infty}, \beta\right)$ is semireflexive and its bidual is $\left(H^{\infty}, \sigma\right)$;

(ii) $\left(H^{\infty}, \beta\right)$ is separable. In fact the sequence $\left\{z \mapsto z^{n}: n=0,1,2, \cdots\right\}$ forms a $(C, 1)$ basis;

(iii) $\left(H^{\infty}, \beta\right)$ cannot be a $(D F)$-space and a fortiori is not quasibarrelled;

(iv) $\beta$ is the topology of uniform convergence on precompact subsets of $\left(H^{\infty}, \beta\right)^{\prime}$ and so is not the Mackey topology.

Proof. We note the following points. (i) This follows immediately from Proposition 1(viii) and Montel's theorem for normal families.

(ii) If $f \in H$ let $s_{n}: z \rightarrow \sum_{k=0}^{n} \alpha_{k} z^{k}$ be the $n$th partial sum of the Taylor expansion of $f$. Let $\sigma_{n}=(1 /(n+1))\left(s_{0}+\cdots+s_{n}\right)$. Then $s_{n} \rightarrow f$ in $\kappa$ and so certainly $\sigma_{n} \rightarrow f$. By Proposition $1(\mathrm{v})$ we need only show that the sequence $\left(\sigma_{n}\right)$ is uniformly bounded. This follows from the usual kernel representation of $\left(\sigma_{n}\right)$. (See, for example [12, Chapter 2], or $[4]$.)

(iii) The author has shown in [8] that a $(D F)$-space in which bounded sets are relatively compact is bornological. Hence if $\left(H^{\infty}, \beta\right)$ were a $(D F)$-space, by (i) we would have $\beta=\sigma$ which is certainly not true.

(iv) By the Corollary of Proposition 2, we need only display a weakly compact subset of $\left(H^{\infty}, \beta\right)^{\prime}$ which is not norm compact. This has been done by Conway in [7]. 


\section{REFERENCES}

1. A. Alexiewicz, On the two-norm convergence, Studia Math. 14 (1953), 49-56. MR 15, 880.

2. R. C. Buck, Operator algebras and dual spaces, Proc. Amer. Math. Soc. 3 (1952), 681-687. MR 14, 290.

3. - Bounded continuous functions on a locally compact space, Michigan Math. J. 5 (1958), 95-104. MR 21 \#4350.

4. - Algebraic properties of classes of analytic functions, Seminar on Analytic Functions, vol. II, Princeton University, Princeton, N. J., 1957, pp. 175-188.

5. H. S. Collins, On the spaces $l^{\infty}(S)$, with the strict topology, Math. Z. 106 (1968), 361-373. MR 39 \#763.

6. H. S. Collins and J. R. Dorroh, Remarks on certain function spaces, Math. Ann. 176 (1968), 157-168. MR 36 \#5694.

7. J. B. Conway, The strict topology and compactness in the space of measures, Trans. Amer. Math. Soc. 126 (1967), 474-486.

8. J. B. Cooper, On a generalization of Silva spaces, Math. Ann. 182 (1969), 309-313.

9. D. J. H. Garling, A generalized form of inductive-limit topology for vector spaces, Proc. London Math. Soc. (3) 14 (1964), 1-28. MR 28 \#4330.

10. A. Grothendieck, Sur les espaces $(F)$ et $(D F)$, Summa Brasil. Math. 3 (1954), 57-123. MR 17, 765.

11. - Espaces vectoriels topologiques, Inst. Mat. Pura Appl., Univ. São Paulo, 1954. MR 17, 1110.

12. K. Hoffman, Banach spaces of analytic functions, Prentice-Hall Series in Modern Analysis, Prentice-Hall, Englewood Cliffs, N. J., 1962. MR 24 \#A2844.

13. D. A. Raykov, A criterion of completeness in locally convex spaces, Uspehi Mat. Nauk 14 (1959), no. 1 (85), 223-229. (Russian) MR 21 \#3747.

14. L. A. Rubel and J. V. Ryff, The bounded weak star topology and the bounded analytic functions, J. Functional Analysis 5 (1970), 167-183.

15. L. A. Rubel and A. L. Shields, The space of bounded analytic functions on a region, Ann. Inst. Fourier (Grenoble) 16 (1966), fasc. 1, 235-277. MR 33 \#6440.

16. H. H. Schaefer, Topological vector spaces, Macmillan, New York, 1966. MR 33 \#1689.

17. A. Wiweger, $A$ topologisation of Saks spaces, Bull. Acad. Polon. Sci. Cl. III. 5 (1957), 773-777. MR 19, 564.

18. - Linear spaces with mixed topology, Studia Math. 20 (1961), 47-68. MR 24 \#A3490.

J. Wolfgang Goethe Universität, Frankfurt-am-Main, West Germany 